American Journal of Applied Sciences 2 (13): 69-77, 2005

ISSN 1546-9239

(C) 2005 Science Publications

\title{
Long Cycles in Employment, Inflation and Real Unit Wage Costs, Qualitative Analysis and Quantitative Assessment
}

\author{
Peter Flaschel, Goeran Kauermann and Timo Teuber \\ Department of Economics and Business Administration, Bielefeld University, Bielefeld Germany
}

\begin{abstract}
This study provided some theoretical foundations for the empirical hypothesis. At first presented two simple prototype models of inflation, stagflation and disinflation. We concluded that there are cycles with a phase length much longer than the ordinary business cycle (in fact approximately five times as long as we saw in the empirical phase plots of the paper) which are not long waves from a Schumpeterian perspective, but caused by the fact that systematic changes in real unit-wage costs and also in inflation caused by changes in the (un-)employment rate may be slow, giving rise to interchanging long-lasting regimes of economic prosperity on the one hand and economic stagnation on the other hand.
\end{abstract}

Key words: Inflation, wage costs, qualitative analysis, quantitative assessment

\section{INTRODUCTION}

In this study we start from evidence on the existence of long clockwise cycles in unemployment rates and inflation on the one hand and in employment rates and the unit wage costs on the other hand, in the U.S. economy after World War II, measured and investigated on the basis of the econometric methodology developed in Kauermann et al. ${ }^{[11]}$ there through an appropriate application to U.S. time series for these three state variables. On the basis of this evidence we develop simple two-dimensional (or simply 2D) macrodynamical models and thereafter more and more integrated modelings of such dynamics that imply by and large such cyclical behavior in employment rates, inflation and the unit wage costs with the observed phase length and amplitude of the two considered long-phased cycles.

The aim of the study is to show that two classical partial modelings of the envisaged cycles can indeed be integrated into more and more coherent wholes without much change in their implications. We thereby finally arrive at a matured, yet conventional type of a 5D Keynesian macrodynamic model that allows for long swings in employment rates, inflation rates and real unit wage costs (or the wage share) of the observed type within a range of parameter values as they are estimated in the empirical study of Chen et $a l .{ }^{[4]}$ of this model type. In this way the study provides theoretical and numerical foundations for the detailed methodological and empirical study undertaken in Kauermann et al. ${ }^{\lceil 11]}$.

In its 2D baseline models the study makes use of perfect anticipations of price inflation without need to employ the rational expectations methodology of the New Keynesian and other macrodynamical schools of thought. In our context this simply helps to save one law of motion (for expectations formation) and allows us to use 2D phase portraits for our baseline theoretical reflection of the observed cyclical behavior. Our final model type of dimension 5 then still relies on such model-consistent expectations (now for both price and wage inflation rates), but couples such perfect short-term expectations with medium-run expectations on a socalled inflation climate surrounding the present evolution of the economy. Here we assume adaptive learning as principle for updating such climate expectations. Assuming myopic perfect foresight with respect to current wage and price inflation again only helps to save further laws of motion (for short-term error correction), while the use of an inflationary climate expression in the employed cost pressure terms of our wage and price Phillips curves adds the necessary inertia to the inflation dynamics investigated in this study. Moreover, this scenario avoids any need for an application of the jumpvariable technique of the rational expectations school, since all variables-though partly forward-looking-can be treated as predetermined ones.

In section 2 of the study we study the empirical phase plot of unemployment rates and price inflation rates (here taken from the empirical investigations performed in Kauermann et $a l^{[11]}$ ) from the partial perspective of the interaction of an accelerationist price Phillips curve with a standard dynamic IS-curve. This interaction is purely explosive in nature unless an active Taylor interest rate policy rule is added to the dynamics, which then-when appropriately chosen-indeed implies slightly convergent dynamics of a long-phased cyclical nature, similar to the shown empirical phase plot, for reasonable choices of the involved parameter values.

In section 3 we then show the same for the phase plot of real unit wage costs against the rate of employment, using now a money wage Phillips curve and a Classical accumulation equation. We thus have at our disposal two partial models that offer theoretical explanations for the two long-phased cycles present in

Corresponding Author: Peter Flaschel, Department of Economics and Business Administration, Bielefeld University, Bielefeld Germany 
the data. Section 4 shows on this basis that there is an easy way to integrate these two partial models into a 3D dynamical system without much change in the generated cycles, which now result from an interaction of a still simple wage-price spiral with a dynamic IScurve and a Classical accumulation equation in the 3D phase space for the considered variables.

In section 5 finally we provide a fully integrated and indeed fairly advanced 5D model of the wage-price spiral, of the dynamics of Keynesian aggregate demand with interest rate as well as distributional effects, using Okun's law as the link between goods and labor markets and a standard type of Taylor interest rate policy rule. This 5D model allows for the same conclusions as the partial 2D and the integrated 3D model. A matured type of conventional Keynesian macrodynamics is therefore able to explain long swings in important economic aggregates as they are observed in the data for the U.S. economy after World War II. We believe that such results are out of reach for the New Keynesian approach to macrodynamics, which is primarily local in nature and-compared to our approachalso by and large trivial in nature in the deterministic part underlying its generally stochastic models with a dynamic IS curve, a New Keynesian Phillips curve and a conventional type of interest rate policy rule.

2D Friedman unemployment/inflation cycles: We start this section by a graphical representation of the unemployment $\mathrm{U}=1$-e/inflation $\pi$ phase plot for the U.S. economy over the period $1955-2004$, i.e., a time span of 50 years (e the rate of employment, to be used in the following theoretical analysis of the depicted situation).

The Fig. 1 shows a single estimated cycle (and its confidence domain) for a period length of approximately 50 years and in addition various subcycles of business cycle frequency. The estimation method used is explained in detail in Kauermann et al. ${ }^{[11]}$ where the obtained results are also considered in much more detail. For the purposes of the present paper we however ignore the shorter cycles and in fact only need the observation that there is evidence for a long-phased overshooting (nonmonotonic) interaction between unemployment and inflation (showing in particular the occurrence of stagflation, i.e., increasing unemployment and increasing inflation) in the U.S. economy after world War II. This clockwise oriented longphased cycle appears to be more complex in situations where unemployment and inflation are both low and is quite regularly structured in the opposite situations.

In order to derive a simple model of such a longphased Friedman cycle in the variables e, the employment rate on the labor market and $\pi=\hat{p}$, the inflation rate on the goods market, we assume a standard (still linear) accelerationist price Phillips curve of a form, as it is for example discussed from the empirical perspective in Stock and Watson ${ }^{[16]}$ (e the NAIRU rate of employment) Eq. (1): $\dot{\pi}=\beta_{\text {pe }}(\mathrm{e}-\overline{\mathrm{e}})$

From the theoretical perspective this equation can be justified through a standard expectations augmented PC, when (adaptively formed) expectations approach the limit situation of myopic perfect foresight. This PPC is here coupled with a dynamic IS-curve-expressed in terms of the employment rate-of the following type ( $\hat{\mathrm{e}}={ }^{\prime} e / e$ the growth rate of the variable) Eq. (2):

$\hat{\mathrm{e}}=-\beta_{\text {uu }}(\mathrm{e}-\overline{\mathrm{e}})-\beta_{\text {ui }}\left(\mathrm{i}-\pi-\left(\mathrm{i}_{\mathrm{o}}-\bar{\pi}\right)\right)$

In this equation we use the postulated equality $e=u$ between the rate of employment $e$ and the rate of capacity utilization $u$ of firms (a simple type of Okun law) and transform through this assumption a goods market adjustment equation into a labor market adjustment equation. The equation assumes that employment rate (capacity utilization) growth or decline depends negatively on the level of the employment (= capacityutilization) rate $e$ (based on the assumption of a marginal propensity to spend of less than one) and also (as usual) negatively on the (perfectly anticipated) actual real rate of interest $i-\hat{\mathrm{p}}$ in its deviation from its steady state value (determined by the steady level of the nominal rate of interest io (which is here given and not yet determined from the real part of the economy in this simple modeling framework) and and the inflation target of the central bank). We stress again that we employ only for the time being an Okun law in the simple form: $e=u$, i.e., changes in the capacity utilization of firms are here reflected in changes of the employment rate by a 1:1 relationship (and assume also $\overline{\mathrm{e}}=\overline{\mathrm{u}}$ for expositional simplicity).

If the nominal interest rate is pegged at its steady state level by the central bank, we get as Jacobian of the considered 2D dynamics in the state variables e, $\pi$ the following qualitative type of matrix:

$$
J=\left(\begin{array}{ll}
0 & + \\
+ & -
\end{array}\right)
$$

i.e., the dynamics are of a saddlepoint type and thus unstable from an historical (predetermined) point of view. We neglect here the jump-variable method of the rational expectations school as a possible solution technique. Instead, we make the dynamical system a stable one by assuming an active, still simple conventional interest rate policy rule of the following simple type:

$$
\mathrm{i}=\mathrm{i}_{\mathrm{o}}-\bar{\pi}+\hat{\mathrm{p}}+\alpha_{\mathrm{p}}(\hat{\mathrm{p}}-\bar{\pi}), \alpha_{\mathrm{p}}>0
$$

This slight simplification of a conventional Taylor rule says that the nominal interest rate it set by the central bank according to its real interest rate target augmented by the current rate of price inflation towards a nominal rate of interest and that the central bank will 
go beyond (stay below) this rate in its interest rate policy if the inflation gap with respect to its inflation target $\bar{\pi}$ is positive (negative). This of course only holds if the parameter $\alpha$ is chosen to be positive, i.e., if the policy is of an active type.

Inserting this rule into the second law of motion gives Eq. (3):

$\hat{\mathrm{e}}=-\beta_{\text {uи }}(\mathrm{e}-\overline{\mathrm{e}})-\beta_{\text {ui }} \alpha_{\mathrm{p}}(\pi-\bar{\pi})$

And thus as new Jacobian matrix:

$$
\mathbf{J}=\left(\begin{array}{cc}
0 & + \\
- & -
\end{array}\right)
$$

According to Olech's Theorem (see Flaschel ${ }^{[8]}$ for its appropriate application when the second law of motion is reformulated in terms of logarithms), these dynamics are globally asymptotically stable in the right half plane of the phase space of the state variables e, $\pi$. Since Goodwin ${ }^{[12]}$ contribution to the analysis of the growth cycle, to be considered in the next section, it is well known from the literature on Lotka-Volterra 2D populations dynamics that their trajectories are everywhere of center type if $\mathbf{J}_{21}=0$ holds true and point inwards with respect to this center dynamics if $\mathrm{J}_{21}<0$ holds and this the stronger the larger this entry becomes in absolute terms. For slightly negative values, i.e., stabilizing dynamic multiplier processes in the goods market dynamics, we therefore get a result as shownin Fig. 2 , i.e., a clockwise rotation in the unemployment-inflation phase space as in the empirical phase plots of these variables shown in Fig. 1. Note that we have now used 1-e in the place of e (as it is standard in the literature) as state variable of the dynamics and that the vertical and horizontal isoclines of these center dynamics have to be employed graphically in order to determine the anticlockwise orientation in $(\mathrm{e}, \pi)$-space and the clockwise rotation in the corresponding $(1-\mathrm{e}, \pi)$ phase space.

Note that the simulated, basically linear (up to the use of growth rates on the left hand side of the equations) dynamics shown in this figure is approximately of the size observed in the postwar evolution of the U.S. economy and has indeed a long phase, since the time span of the shown simulation is also 50 years. We have used (for annualized data) crude OLS estimates in determining the approximate size of the parameters of the dynamics (1), (3) in order to relate this simple dynamical model to real world data, yet have chosen an inflation target of $\bar{\pi}=0.06$ in order to avoid the occurrence of deflation (The employed parameter values are: $\beta_{\mathrm{pe}}=0.2 ; \overline{\mathrm{e}}=0.9 ; \beta_{\mathrm{ui}} \alpha \mathrm{p}=0.1$; $\left.\overline{\mathrm{u}}=0.06 ; \beta_{\mathrm{uu}}=0.01\right)$. We learn from this very simple approach to an indeed keynesian representation of Friedman type inflation dynamics that the private sector is here not at all viable by itself, but crucially needs an active interest rate policy in order to create stable dynamics, turned into long-phased asymptotically stable dynamics through the negative eigen-feedback that exists in the second law of motion. A simple combination of a current type of Phillips curve with a standard dynamic IS-relationship and a Taylor type policy rule of current textbook type is thus already sufficient for providing a reasonable model of the long-phased cycle observed for the interaction of unemployment and inflation in the U.S. economy after World War II.

2D Goodwin employment/income distribution cycles: As in the preceding section we start in this section from a graphical representation now of the employment e/wage share $v$ phase plot for the U.S. economy again over the period 1955-2004, i.e., again a time span of 50 years.

This figure shows again a single estimated cycle (and its confidence domain) for a period length of approximately 50 years and again various subcycles of business cycle frequency. We again ignore the shorter cycles and in fact only need the observation that there is, here too, evidence for a long-phased overshooting (non-monotonic) interaction now between the employment rate $e$ and the share of wages $v$ in national income. This againclockwise oriented long-phased cycle appears to be more complex in situations of a high employment rate and is relatively simple structured in the opposite situations.

In order to present again a simple model of such a long-phased accumulation cycle in the variables $e$ and $v$ we make use of seminal papers of both Goodwin ${ }^{[12]}$ and Rose $^{[14]}$. From this perspective, the envisaged cyclegenerating feedback structure can be based on the following two laws of motion Eq. (5):

$$
\begin{aligned}
& \hat{v}=\dot{v} / v=\beta_{v e}(e-\bar{e})-\beta_{v v}(v-\bar{v}) \\
& \bar{e}=\dot{e} / e=-\beta_{e v}(v-\bar{v})
\end{aligned}
$$

where, $\mathrm{v}$ denotes real unit-wage costs (or the share of wages in GDP). We assume again for the time being that $\mathrm{e}$ $=\mathrm{u}$ holds and can e.g., justify Eq. (4) by means of the wage dynamics investigated in Blanchard and Katz ${ }^{[3]}$, with perfect anticipation of price inflation now (implying a real wage Phillips curve) where in addition to demand pressure we have unit wage costs acting as an error correction mechanism on their own evolution (We neglect the use of logarithms here and elsewhere for reasons of simplicity, see Chen et al. ${ }^{[4]}$ for their inclusion and motivation and for a general theory of such a real wage or wage share PC (when labor productivity is assumed a given magnitude)). In the second law of motion we now focus on a goods market behavior that is profit-led, i.e., increases in unit wage costs act negatively on aggregate demand and thus negatively on the growth rate of the rate of capacity utilization $\mathrm{u}(=\mathrm{e}$, the rate of employment). We abstract from the real rate of interest effect and from the dynamic multiplier process considered in the preceding section in this baseline model of long-phased cyclical growth. 
If $\beta_{\mathrm{vv}}=0$ holds, as Blanchard and Katz assert it for the U.S. economy, we have the same cross-dual dynamics of the Goodwin ${ }^{[12]}$ growth cycle model and thus again a center type dynamics that is stable, but not asymptotically stable. In the case $\beta_{\mathrm{vv}}>0$ we can again apply Olech's Theorem (in the same way as for the Friedman cycle model) and obtain from it global asymptotic stability of the dynamics in the positive orthant of the phase plane with respect to the uniquely determined interior steady state position $\overline{\mathrm{e}}, \overline{\mathrm{v}}$. For weak Blanchard and $\mathrm{Katz}^{[3]}$ error correction terms we thus get a somewhat damped longphased cyclical motion in the wage share/employment rate phase space as shown in Fig. 4. As in the previous model for the unemployment and inflation rate interaction, we get a clockwise rotation in this new phase space with approximately one cycle in 50 years (9The parameters underlying this simulation are: $\beta_{\mathrm{ve}}=0.06 ; \overline{\mathrm{e}}=$ $0.9 ; \beta_{\mathrm{vv}}=0.01 ; \beta_{\mathrm{ev}}=0.1 ; \overline{\mathrm{v}}=0.6$. and are again approximately obtained from simple OLS estimates of these dynamics (with no good statistical properties however, but definitely more appropriately chosen compared to the case without any empirical reference)).

In the next section we shall provide a simple integration of the nominal and the real dynamics considered in the last and in this section. It will show that the two long-phased cycle generators are in fact just two sides of the same coin and are thus fairly closely related to each other.

Friedman and Goodwin cycles in 3D interaction: We have shown that two simple approaches to price and wage Phillips curves, augmented by a goods market dynamics of Keynesian IS-type in the first case and by a growth law of the capital stock and the employment rate in the second case, produce two types of long-phased cycles in inflation and in income distribution, both of a prototype that is well-known from the literature as far as building blocks, yet not necessarily as far as implications are concerned. These two cycle mechanism can however easily integrated into a coherent whole by assuming the following integrated form of goods market dynamics as link between the considered inflation rate and the wage share dynamics Eq. (6-8):

$$
\begin{aligned}
& \dot{\pi}=\beta_{\text {pe }}(\mathrm{e}-\overline{\mathrm{e}}) \\
& \hat{\mathrm{e}}=-\beta_{\text {uu }}(\mathrm{e}-\overline{\mathrm{e}})-\beta_{\text {ui }}\left(\mathrm{i}-\pi-\left(\mathrm{i}_{\mathrm{o}}-\bar{\pi}\right)\right)-\beta_{\text {eu }}(v-\bar{v}) \\
& v=\beta_{v e}(\mathrm{e}-\overline{\mathrm{e}})-\beta_{v v}(v-\bar{v})
\end{aligned}
$$

Which in a minimal way connects the PPC dynamics of Friedmanian type with the WPC dynamics of Goodwinian type. The Jacobian of these integrated dynamics in the state variables $v, e, \pi$ reads:

$$
\mathrm{J}\left(\begin{array}{lll}
0 & + & 0 \\
- & - & - \\
0 & + & 0
\end{array}\right)
$$

It can be easily shown to be locally asymptotically stable by means of the Routh-Hurwitz stability conditions. Global results are however no longer so easily available as in the preceding sections, though they will hold as before due to the simple nonlinear form of the considered dynamics.

The result of this coupling of two cycle mechanisms is shown in Fig. 5, top left. We see from this figure that the two cycles just integrate themselves into a simple 3D cycle and thus do not produce anything significantly new with respect to their isolated operation. This is also motivated further by Fig. 5, bottom right, where we see that inflation and real unit-wage costs are strictly positively related along the generated cycle. One can conclude from this that these two cycle mechanisms are fairly synchronized with each other and thus do not give rise to complex oscillation as they can be in principle obtained from such coupled oscillators.

The result of this section is that we can indeed view the inflation dynamics and the real wage or wage share dynamics as being closely relate with each other and worthwhile to be considered from a more general perspective as it has been developed in Chen et al.[4]. There an advanced type of wage-price spiral, the DAS side of the model, is coupled with an advanced description of the dynamics of aggregate demand, the DAD side of the model, in order to provide a matured type of conventional Keynesian macrodynamics as an alternative to the New Keynesian macrodynamics with both staggered wage and price settings. The question then is whether such an updated or contemporaneous type of conventional Keynesian macrodynamics will also reproduce the cycles we have considered in this study so far.

5 Long-phased inflation and distribution cycles in an advanced Keynesian macro model: In this section we modify the disequilibrium model of AS-AD growth of Asada et al. ${ }^{[2]}$ and its empirical reformulation in Chen et $a l .^{[4]}$. in order to obtain a model version that integrates the results of the preceding sections into a framework that can be considered as complete from the perspective of Keynesian disequilibrium AS-AD macromodels as they were introduced in these two papers. We now make use of an integrated dynamic IS equation coupled with an employment dynamic, representing goods market dynamics as before and on this basis the link between goods and labor markets as provided by Okun ${ }^{[13]}$. We also assume again a conventional type of Taylor interest rate policy rule, now with interest smoothing in addition.

As far as the supply side of the considered macroeconomy is concerned we make use of an advanced model of the wage-price spiral as it was introduced in Chiarella et al. ${ }^{[7]}$ and further considered in the above mentioned papers. We thus now use-besides labor and goods market pressure terms in the wage and the price Phillips curve shown below-Blanchard and $\mathrm{Katz}^{[3]}$ error correction terms in both of these Phillips curves and thus give income distribution a role to play in wage as well as in price dynamics. Finally, we add inflationary inertia to 
the world of myopic perfect foresight of the preceding sections (with neoclassical dating of inflationary expectations), through the inclusion of a medium-run variable, the inflationary climate $\pi^{\mathrm{c}}$ in which the economy is operating. We use this climate variable as well as model-consistent expectations of wage and price inflation in order to define wage cost pressure and price cost pressure in the price and the wage Phillips curve as a weighted average of model-consistent wage and price inflation rates (based on myopic perfect foresight) and this climate expression, respectively.

We thus now consider the following type of a crossover dynamic interaction between wage and price inflation rates $\overline{\mathrm{w}}, \overline{\mathrm{p}}$ in the place of our earlier simplified wage and price Phillips curves:

$$
\begin{aligned}
& \hat{w}=\beta_{w e}(e-\bar{e})-\beta_{w v}(v-\bar{v})+\kappa_{w} \hat{p}+\left(1-\kappa_{w}\right) \pi^{c}+n_{x}, \\
& \hat{p}=\beta_{p u}(u-\bar{u})+\beta_{p u}(v-\bar{v})+\kappa_{p}\left(\hat{w}-n_{x}\right)+\left(1-\kappa_{p}\right) \pi^{c}
\end{aligned}
$$

Regarding productivity growth we assume Harrodneutral technical change with a given rate $n_{x}=\hat{x}=$ const. This technological background of our model has been integrated into the above wage-price spiral such that it allows for a steady state solution.

Somewhat simplified versions of these two Phillips curves have been estimated for the U.S. economy in various ways in Flaschel and Krolzig ${ }^{[9]}$, Flaschel et $a l^{[11]}$ and Chen and Flaschel ${ }^{[6]}$ and have been found to represent a significant improvement over conventional single reduced-form Phillips curves. A particular finding was that wage flexibility was greater than price flexibility with respect to demand pressure measures in the market for goods and for labor, respectively and that workers are more short-sighted than firms with respect to their cost pressure terms. Note thatsuch a finding is not possible in the conventional framework of a single reduced-form Phillips curve.

Inflationary expectations over the medium run, $\pi^{c}$, i.e., the inflationary climate in which current perfectly foreseen inflation rates are operating, may be adaptively following the actual rate of price inflation (by use of some linear or exponential weighting scheme), may be based on a rolling sample (with hump-shaped weighting schemes), or on other possibilities for updating such a climate expression. For simplicity of the exposition we shall make use here of a conventional adaptive mechanism, namely:

$$
\dot{\pi}^{\mathrm{c}}=\beta_{\pi \mathrm{c}}\left(\hat{\mathrm{p}}-\pi^{\mathrm{c}}\right)
$$

Note that in this version of the wage-price spiral, the inflationary climate variable does not matter for the evolution of the wage share $v=\omega / \mathrm{x}=\mathrm{wL} / \mathrm{pY}$, the law of motion of which is given by (with $\kappa=1 /\left(1-\kappa_{\mathrm{w}} \kappa_{\mathrm{p}}\right)$ ):

$$
\hat{v}=\kappa\left[\begin{array}{l}
\left(1-\kappa_{p}\right)\left(\beta_{w e}(e-\bar{e})-\beta_{w v} \mid(v-\bar{v})\right) \\
-\left(1-\kappa_{w}\right)\left(\beta_{p u}(u-\bar{u})+\beta_{p u}(v-\bar{v})\right)
\end{array}\right]
$$

This follows easily from the following reduced-form or across-market representation of the above two PC's:

$$
\begin{aligned}
& \hat{\mathrm{w}}=\kappa\left[\begin{array}{l}
\left.\beta_{\mathrm{we}}(\mathrm{e}-\overline{\mathrm{e}})-\beta_{\mathrm{wu}}(v-\bar{v})\right) \\
-+\kappa_{\mathrm{w}}\left(\beta_{\mathrm{pu}}(\mathrm{u}-\overline{\mathrm{u}})+\beta_{\mathrm{pu}}(v-\bar{v})\right)
\end{array}\right]+\pi^{\mathrm{c}}, \\
& \hat{\mathrm{p}}=\kappa\left[\begin{array}{l}
\beta_{\mathrm{pu}}(\mathrm{u}-\overline{\mathrm{u}})+\beta_{\mathrm{pv}}(v-\bar{v}) \\
+\kappa_{\mathrm{p}}\left(\beta_{\mathrm{we}}(\mathrm{e}-\overline{\mathrm{e}})-\beta_{\mathrm{wv}}(v-v)\right)
\end{array}\right]+\pi^{\mathrm{c}}
\end{aligned}
$$

Which represent a considerable generalization of the conventional view of a single-market reduced-form price PC with only one measure of demand pressure, the one in the labor market. In view of the opposite signs for the influence of labor market pressure e-ē and goods market pressure $\mathrm{u}-\overline{\mathrm{u}}$, we say that wage share dynamic is labormarket led if the wage share increases with economic activity and goods-market led in the opposite case. Note that such statements depend on the link between labor and goods market activity levels as it will be supplied below and that we have assumed wage share dynamics to be labor market-led in the preceding sections,

The remaining laws of motion of the private sector of the model are as follows:

$$
\begin{aligned}
& \hat{\mathrm{u}}=-\beta_{\text {uu }}(\mathrm{u}-\overline{\mathrm{u}}) \pm \beta_{\mathrm{uv}}\left(v-v_{\mathrm{o}}\right)-\beta_{\text {ui }}\left((\mathrm{i}-\hat{\mathrm{p}})-\left(\mathrm{i}_{\mathrm{o}}-\bar{\pi}\right)\right), \\
& \hat{\mathrm{e}}=\text { bû or more specific } \frac{\mathrm{e}}{\overline{\mathrm{e}}}=\left(\frac{\mathrm{u}}{\overline{\mathrm{u}}}\right)^{\mathrm{b}}
\end{aligned}
$$

The first law of motion is again of the type of a dynamic IS-equation, represented by the growth rate of the capacity utilization rate of firms and is of the same form as used for our integrated dynamics in the preceding section. It has three important characteristics; (i) it reflects the dependence of output changes on aggregate income and thus on the rate of capacity utilization by assuming a negative, i.e., stable dynamic multiplier relationship in this respect, (ii) it shows the joint dependence of consumption and investment on the wage share or real unit-wage costs (which in the aggregate may in principle allow forpositive or negative signs before the parameter $\alpha v$, depending on whether consumption or investment is more responsive to wage-share or wage-cost changes) and (iii) it shows finally the negative influence of the real rate of interest on the evolution of economic activity. Note here that we have generalized this law of motion slightly in comparison to the one used in the preceding subsection. We call goodsmarket dynamics wage-led if it depends positively on the share of wages in GDP and profit-led in the opposite case. In view of this terminology, we have only considered profit-led economies in the preceding sections.

In the second law of motion, for the rate of employment, we assume that the employment policy of firms follows-in the form of an Okun Law-the rate of capacity utilization (and the thereby implied rate of over-or underemployment of the employed workforce) 
only to certain degree (through a positive parameter $b$ ). Employment is thus assumed to adjust to the level of current activity in a form as it was originally specified by Okun ${ }^{[13]}$ himself (whereby we replace our simple identification $e=u$ of the preceding sections).

As interest rate policy we finally assume the following classical type of a Taylor rule:

$$
\begin{aligned}
& i^{*}=\left(i_{o}-\bar{\pi}\right)+\hat{p}+\alpha_{p}(\hat{p}-\bar{\pi}) \\
& i=\alpha_{i}\left(i^{*}-i\right)
\end{aligned}
$$

The target rate of the central bank $i^{*}$ is here made dependent on the steady state real rate of interest augmented by actual inflation rate back towards a nominal rate and it is as usually dependent on the inflation gap with respect to inflationary target $\bar{\pi}$. With respect to this target there is then interest rate smoothing with strength $\alpha_{i}$.

The steady state of the dynamics (given by the exogenous parameters of the model) is locally asymptotically stable under certain sluggishness conditions that are reasonable from a Keynesian perspective, loses its asymptotic stability cyclically (by way of socalled Hopf-bifurcations) if the system becomes too flexible and becomes sooner or later globally unstable if (generally speaking) adjustment speeds become too high, see Chen et al. ${ }^{[4]}$ for details. If the model is subject to explosive forces, it requires extrinsic nonlinearities in economic behavior-like downward money wage rigidity-to manifest themselves at least far off the steady state in order to bound the dynamics to an economically meaningful domain in the considered 5D state space. Chen et al. ${ }^{[5]}$ provide a variety of numerical studies for such an approach with extrinsically motivated nonlinearities and thus undertake its detailed numerical investigation. In sum, therefore, our dynamic disequilibrium AS-AD growth model here and there will exhibit a variety of features that are much more in line with a Keynesian understanding of the characteristics of the business cycle as well as longer ones than is the case for the conventional modeling of AS$\mathrm{AD}$ growth dynamics or its radical reformulation by the New Keynesians (where-if non-determinacy can be avoided by the choice of an appropriate Taylor rule-only the steady state position is generally a meaningful solution in a deterministic setup, see Chiarella et $_{\text {al }}{ }^{[7]}$ for details.

Taken together the model of this subsection consists of the following five laws of motion Eq. (9-13):

$$
\begin{aligned}
& \hat{\mathrm{u}}=-\beta_{\text {uu }}(\mathrm{u}-\overline{\mathrm{u}}) \pm \beta_{\mathrm{uv}}(v-\overline{\mathrm{v}})-\beta_{\text {ui }}\left((\mathrm{i}-\hat{\mathrm{p}})-\left(\mathrm{i}_{\mathrm{o}}-\bar{\pi}\right)\right) \\
& \hat{\mathrm{e}}=\text { bû or more specific } \frac{\mathrm{e}}{\overline{\mathrm{e}}}=\left(\frac{\mathrm{u}}{\overline{\mathrm{u}}}\right)^{\mathrm{b}} \\
& \mathrm{i}=\alpha_{\mathrm{i}}\left(\mathrm{i}_{\mathrm{o}}-\bar{\pi}+\hat{\mathrm{p}}+\alpha_{\mathrm{p}}(\hat{\mathrm{p}}-\bar{\pi})-\mathrm{i}\right)
\end{aligned}
$$
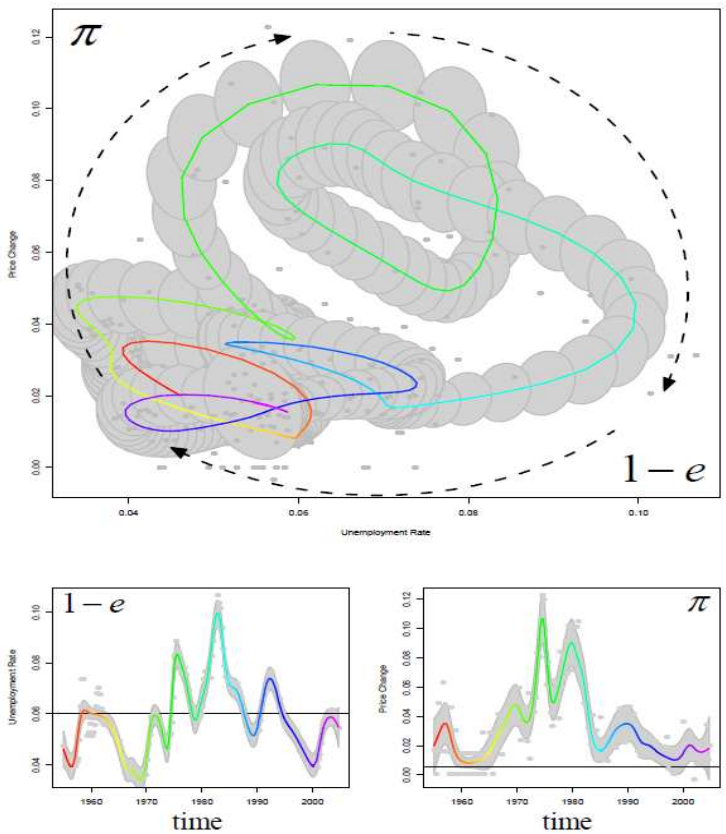

Fig. 1: Friedmanian unemployment/inflation dynamics

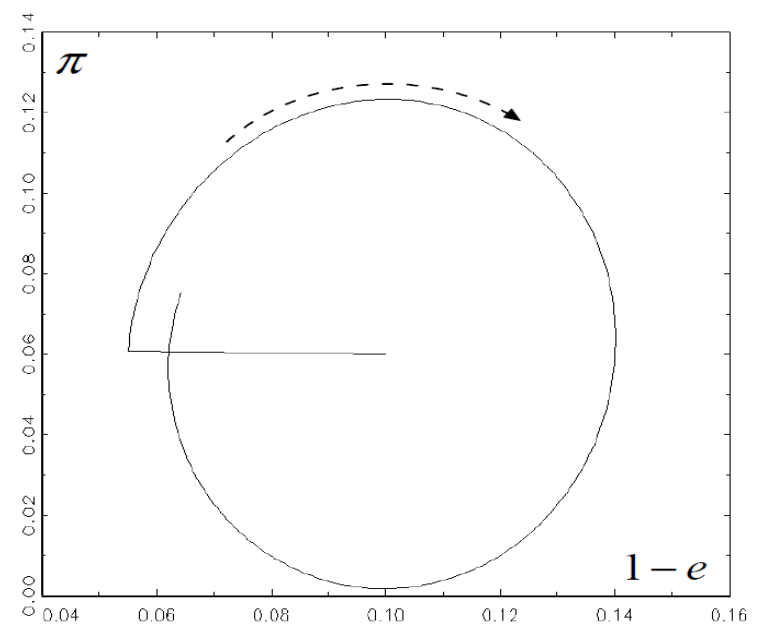

Fig. 2: Friedman type long-phased unemployment/inflation dynamics

$\bar{v}=\kappa\left[\begin{array}{l}\left(1-\kappa_{\mathrm{p}}\right)\left(\beta_{\mathrm{we}}(\mathrm{e}-\overline{\mathrm{e}})-\beta_{\mathrm{wv}}(v-\bar{v})\right) \\ -\left(1-\kappa_{\mathrm{w}}\right)\left(\beta_{\mathrm{pu}}(\mathrm{u}-\overline{\mathrm{u}})+\beta_{\mathrm{pv}}(v-\bar{v})\right)\end{array}\right]$

$\dot{\pi}^{\mathrm{c}}=\beta_{\pi^{c}}\left(\overline{\mathrm{p}}-\pi^{\mathrm{c}}\right)$

The above equations represent, in direct comparison to the baseline model of New Keynesian macroeconomics, the IS goods market dynamics, here augmented by Okun's Law as link between the goods and the labor market and of course the Taylor Rule and 
an advanced law of motion for the wage share that makes use of the same explaining variables as the New Keynesian one, yet with no sign reversal when reduced form expressions are formed and finally the law of motion that describes the updating of the inflationary climate expression. We have to make use in addition of the following reduced-form expression for the price inflation rate in three of the above laws of motion in order to arrive at an autonomous dynamical system Eq. (14):

$$
\hat{\mathrm{p}}=\kappa\left[\begin{array}{l}
\beta_{\mathrm{pu}}(\mathrm{u}-\overline{\mathrm{u}})+\beta_{\mathrm{p} v}(v-\bar{v}) \\
+\kappa_{\mathrm{p}}\left(\beta_{\mathrm{we}}(\mathrm{e}-\overline{\mathrm{e}})-\beta_{\mathrm{wv}}(\mathrm{v}-\overline{\mathrm{v}})\right)
\end{array}\right]+\pi^{\mathrm{c}}
$$

The dynamics are shown to be locally asymptotically stable if estimated signs (not sizes) of the parameter values of the 5D dynamics are employed and if the inflationary climate is updated sufficiently sluggishly. In the estimated situation the labor market dominates the law of motion of the wage share (which is therefore labor market led) and there is a negative impact effect of the wage share on the goods market dynamics (which are therefore profit led, as in the simple Goodwin model of the growth cycle, see Chen et al. ${ }^{[4]}$ for details). Increasing the adjustment speed of the inflationary climate expression however implies (sooner or later) loss of stability by way of a Hopf-bifurcation and basically explosive behavior beyond this Hopf-bifurcation point.
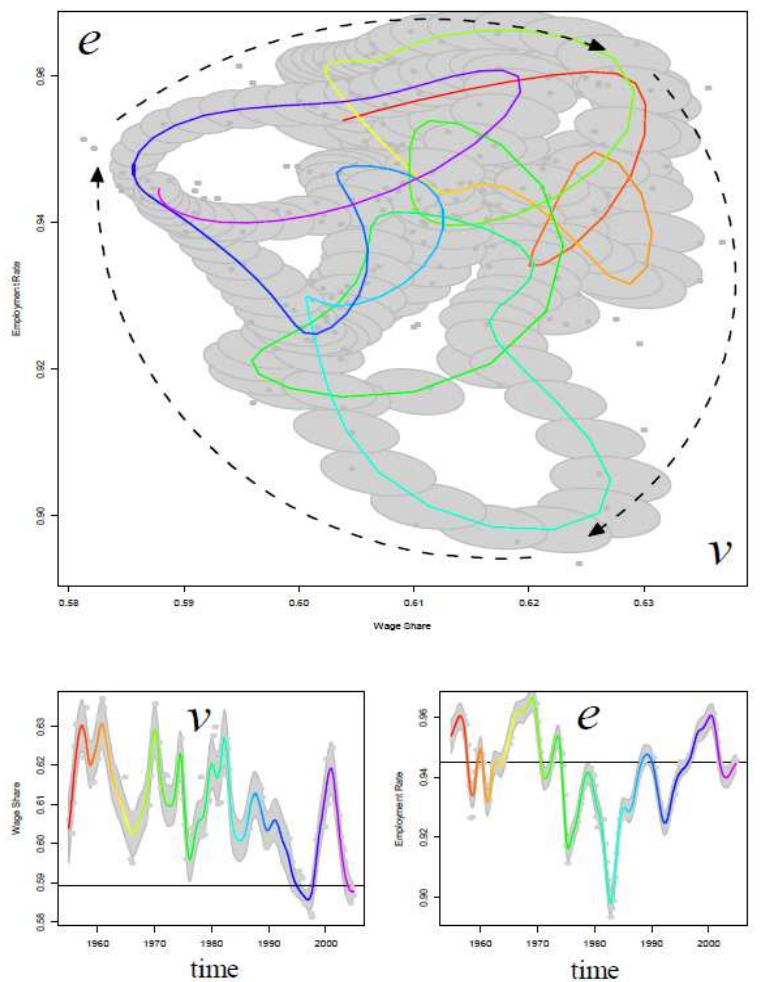

Fig. 3: Goodwinian wage share/employment rate dynamics
Behavioral nonlinearities have then to be employed in order to keep the dynamics bounded and economically viable, see Chen et al. ${ }^{[5]}$ for details.

We here simulate the implied 5D dynamics (or 4D dynamics if Okun's Law is used in level form) with the following parameter values, values that are by and large of the same size to the ones estimated for this model type in Chen et al. ${ }^{[4]}$ :

$$
\begin{aligned}
& \beta_{\text {wu }}=0.9 ; \overline{\mathrm{e}}=0.945 ; \beta_{\mathrm{wv}}=0.004 ; \kappa_{\mathrm{w}}=0.6 \\
& \beta_{\mathrm{pu}}=0.12 ; \overline{\mathrm{u}}=0.82 ; \beta_{\mathrm{pv}}=0 ; \kappa_{\mathrm{p}}=0.4 ; \\
& \beta_{\mathrm{uu}}=0.002 ; \beta_{\mathrm{ui}} \alpha_{\mathrm{p}} \alpha_{\mathrm{i}}=0.2 ; \beta_{\mathrm{uv}}=0.2 \\
& \bar{\pi}=0.03 ; \bar{v}=0.6 ; \mathrm{b}=0.3 ; \beta_{\pi^{c}}=0.38
\end{aligned}
$$

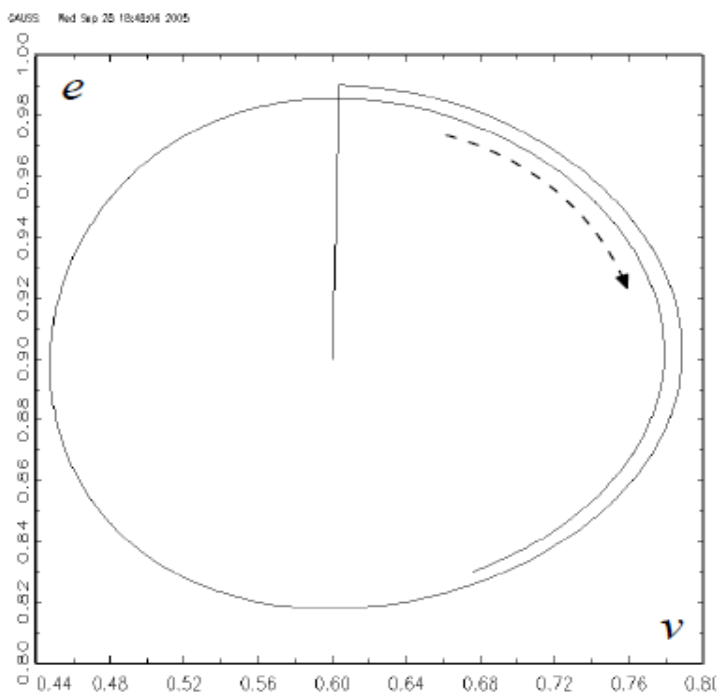

Fig. 4: Goodwin-type long-phased wage share/employment dynamics
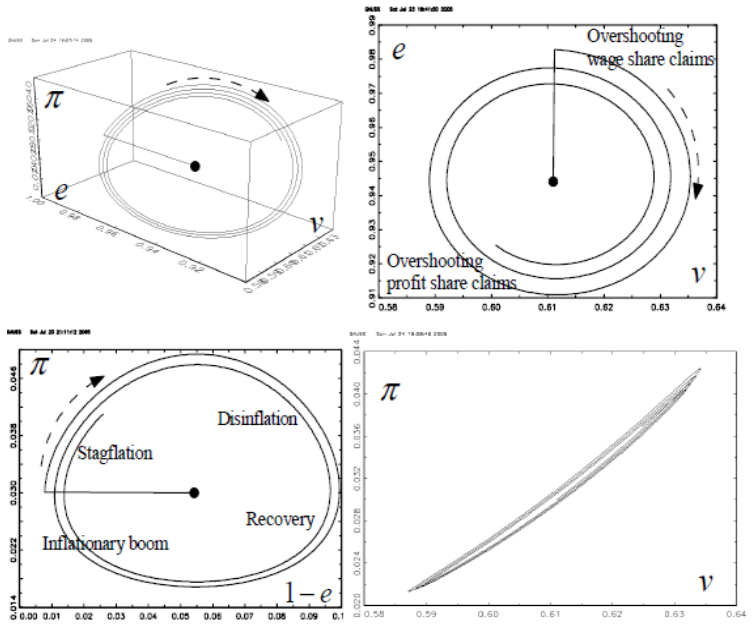

Fig. 5: Friedman inflation and Goodwin wage share dynamics and their interaction 

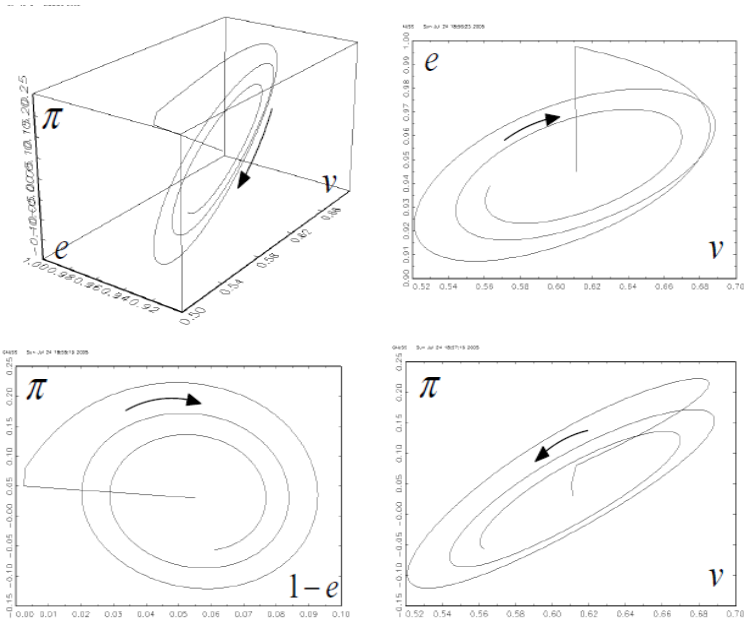

Fig. 6: Goodwin and Friedman cycles in a full-fledged disequilibrium AS-AD model
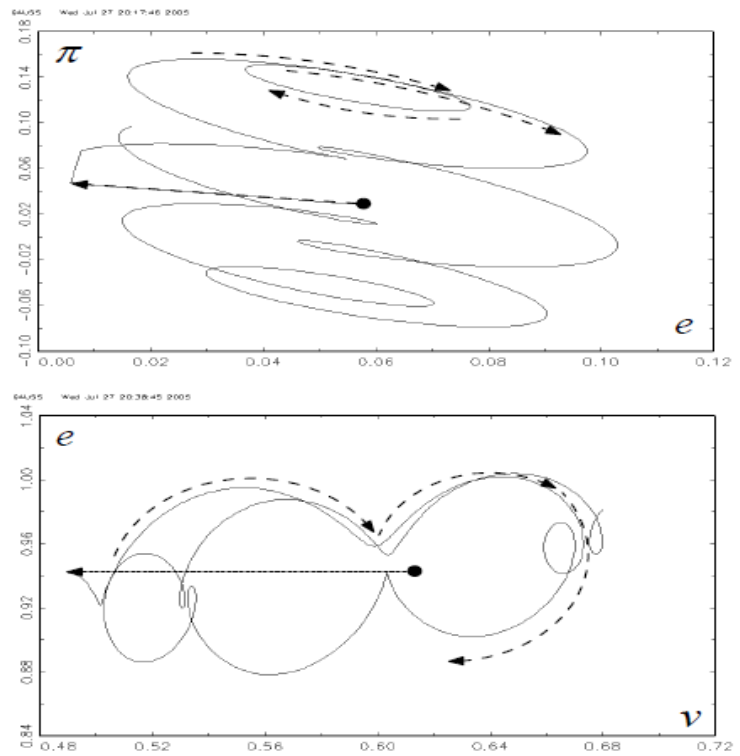

Fig. 7: Goodwin and Friedman cycles and the business cycle

These values again imply that the dynamics of real unit-wage costs is labor-market led (these costs are therefore increasing with an increase in economic activity) and thatthe goods-market dynamics are profitled, i.e., the growth rate in capacity utilization depends negatively on real unit-wage costs. We have furthermore a weak stabilizing influence from economic activity on its rate of growth (a stable dynamic multiplier) and from the wage share onto its rate of growth (through Blanchard and Katz error correction in the labor market). Finally, we have that monetary policy is sufficiently active in order to turn the destabilizing real rate of interest effect into a stabilizing one, i.e., the influence of an increasing rate of inflation on economic activity is tamed as in the basic Friedman cycle model. Concerning the speed of adjustment $\beta_{\pi}^{c}$ of the inflationary climate towards the behavior of actual inflation we however assume that this parameter can be considered as a free parameter in our model and have set this parameter to a value that gives rise to mildly damped oscillations, as they were observed in the two simpler (partial) models considered in the preceding sections.

The Fig. 6 shows a simulation run for the now considered stage of the modeling process, where the two cycles of the preceding section again interact, now with a proper formulation of Okun's Law linking the goods and the labor market and a much more advanced formulation of the interaction of wage and price inflation, particularly in view of the inflationary climate expression that is here used to provide sufficient inertia in the considered dynamics.

The here considered fully integrated model type (9)(13) generalizes significantly the models of the preceding sections without changing the dynamic implications of these models very much. It can be shown to be asymptotically stable, see Chen et al. ${ }^{[4]}$, if the signs of the parameters are as assumed above (independent of their size), if in addition the speed parameter $\beta_{\pi}^{c}$ is chosen sufficiently low. The simple cycle shown in the 3D projection is basically of the form as it was obtained for the integrated Friedman-Goodwin cycle of the preceding section, though a bit rotated compared to the one in Fig. 5. The employment-rate wage-share plot has become a bit procyclical, while the plot of inflation against the wage share is now less procyclical. The inflation unemployment phase plot is however of nearly the same shape as before. The two cycle mechanisms discussed in the preceding sections are again fairly synchronized and suggest again that the Goodwin and the Friedman dynamic are indeed fairly closely related to each other.

The time interval underlying the simulation run is again 50 years, i.e., the phase length of the cycle is close to the ones of the preceding sections. We stress that the model is based on constant parameters and thus not close to what may generate the U.S. business cycle and its phase length of approximately 8 years. Such cycles may be generated through varying propensities to consume, to invest and also varying conditions on the financial markets of the economy (that are here all excluded here from consideration). Instead we have focused on long run movements in the rate of employment, real unit-wage costs and the rate of inflation and thereby generated economic fluctuations in these expressions that appear to be five times as long as the business cycle in the U.S economy as an inspection of the empirical counterparts to the above phase plots seems to suggest (Fig. 1 and 3). This however is a topic that must be investigated in much more detail and with much more care in future research and will also be the focus of interest in the study by Kauermann et al. ${ }^{[11]}$. 
Before we close this section we add a simulation run where a sin-wave representing U.S. business cycle frequency has been added to the simulation shown in Fig. 6 with respect to the evolution of the utilization rate $u$. Fig. 7 shows with respect to the Friedman inflation and the Goodwin wage share cycles the result of such an addition which indicates that the clockwise loops investigated in these section from the theoretical perspective may become quite involved if such a coupling of cycles of different frequencies occurs.

In closing this section we also note that a significantly larger parameter $\beta_{\mathrm{p}}{ }^{\mathrm{u}}$ can reverse the orientation in the cycles shown in Fig. 6,7 and that a stronger (negative) real rate of interest effect in the goods market dynamics need not improve the stability features of the dynamics. Finally, making interest rate policy more active with respect to the inflation gap need not improve the stability of the system, but can make it more volatile and even explosive. Changing parameters may therefore not imply stability result in line with what would be expected from partial macrodynamic reasoning.

\section{CONCLUSION}

In this study we have provided some theoretical foundations for the empirical hypothesis that there exist clockwise-oriented long-phased fluctuations in the core variables unemployment rate, 1-e and inflation rate, $\pi$, on the one hand and employment rate e and real unitwage costs, v, on the other hand.

We have at first presented two simple prototype models of inflation, stagflation and disinflation in the spirit of Friedman's reflection of such issues and of employment and real unit-wage costs (or the wage share) in the spirit of Goodwin $^{[12]}$ growth cycle model. It has already been observed by Atkinson ${ }^{[1]}$ that in particular models of the Goodwin type tend towards long-phased fluctuations in employment and the wage share for reasonable parameter values. It may however be somewhat surprising that the same holds true (for reasonable sizes of the parameter in the employed Phillips curve) for the employment rate/inflation cycle as well, a cycle mechanism that is formally identical to the Goodwin one as we have shown.

We thus arrived at the conclusion that there are cycles with a phase length much longer than the ordinary business cycle (in fact approximately five times as long as we saw in the empirical phase plots of the study) which are not long waves from a Schumpeterian perspective, but caused by the fact that systematic changes in real unit-wage costs and also in inflation caused by changes in the (un-) employment rate may be slow, giving rise to interchanging longlasting regimes of economic prosperity on the one hand and economic stagnation on the other hand.

\section{REFERENCES}

1. Atkinson, A.B., 1969. The time-scale of economic models: How long is the long run? Rev. Econom. Stud., 36: 137-152.
2. Asada, T., P. Chen, C. Chiarella and P. Flaschel, 2005. AD-AS and the Phillips curve. A baseline disequilibrium approach. In: Quantitative and Empirical Analysis of Nonlinear Dynamic Macromodels, Chiarella, C., P. Flaschel, R. Franke and W. Semmler, (Eds.), Elsevier, Amsterdam,

3. Blanchard, O.J. and L. Katz, 1999. Wage dynamics: Reconciling theory and evidence. Am. Econom. Rev. Papers Proc., 1: 69-74.

4. Chen, P., C. Chiarella, P. Flaschel and W. Semmler, 2005. Keynesian macrodynamics and the Phillips curve: An estimated baseline macro-model for the U.S. economy. In: Quantitative and Empirical Analysis of Nonlinear Dynamic Macromodels, Chiarella, C., P. Flaschel, R. Franke and W. Semmler, (Eds.), Elsevier, Amsterdam.

5. Chen, P., C. Chiarella, P. Flaschel and H. Hung, 2005. Keynesian disequilibrium dynamics: Estimated convergence, roads to instability and the emergence of complex business fluctuations. In: Advances in Macroeconometric Modeling, Galler, H. and C. Dreger, (Eds.), Baden-Baden: Nomos Verlagsgesellschaft.

6. Chen, P. and P. Flaschel, 2005. Measuring interacting wage and price Phillips curves for the U.S. economy.

7. Chiarella, C., P. Flaschel and R. Franke, 2005. Foundations for a Disequilibrium Theory of the Business Cycle. Qualitative Analysis and Quantitative Assessment. 1st Edn., Cambridge, UK: Cambridge University Press.

8. Flaschel, P., 1984. Some stability properties of Goodwin's growth cycle. A critical elaboration. J. Econom., 44: 63-69.

9. Flaschel, P. and H.M. Krolzig, 2005. Wage-price Phillips curves and macroeconomic stability: Basic structural form, estimation and analysis. In: Quantitative and Empirical Analysis of Nonlinear Dynamic Macromodels, Chiarella, C., P. Flaschel, R. Franke and W. Semmler, (Eds.), Elsevier, Amsterdam.

10. Flaschel, P., G. Kauermann and W. Semmler, 2005. Testing wage and price Phillips curves for the United States. Metroeconomica, forthcoming.

11. Kauermann, G., P. Flaschel and T. Teuber, 2005. Estimating long-phased cycles in inflation and real unit-wage costs using penalized splines. Sydney: University of New South Wales.

12. Goodwin, R.M., 1967. A growth cycle. In: Socialism, Capitalism and Economic Growth, Feinstein, C.H., (Ed.), Cambridge, UK: Cambridge University Press, pp: 54-58.

13. Okun, A.M., 1970. The Political Economy of Prosperity. Washington, D.C., (Ed.), The Brookings Institution.

14. Rose, H., 1967. On the non-linear theory of the employment cycle. Rev. Econom. Stud., 34: 153-173.

15. Stock, J.H. and M.W. Watson, 1999. Business fluctuations in US macroeconomic time series. In: Handbook of Macroeconomics, Taylor, J.B. and M. Woodford, (Eds.), Amsterdam, Elsevier. 